\title{
ANTIMAGIC LABELING OF GENERALIZED SAUSAGE GRAPHS
}

\author{
Oudone Phanalasy \\ School of Mathematical and Physical Sciences \\ University of Newcastle, Australia \\ and \\ Department of Mathematics \\ National University of Laos, Laos \\ oudone.phanalasy@gmail.com
}

\begin{abstract}
An antimagic labeling of a graph with $q$ edges is a bijection from the set of edges to the set of positive integers $\{1,2, \ldots, q\}$ such that all vertex weights are pairwise distinct, where the vertex weight of a vertex is the sum of the labels of all the edges incident with that vertex. A graph is antimagic if it has an antimagic labeling. In this paper we construct antimagic labeling for the family of generalized sausage graphs.
\end{abstract}

Key words: Antimagic labeling, generalized sausage graph.

\begin{abstract}
Abstrak. Sebuah pelabelan anti-ajaib dari sebuah graf dengan $q$ sisi adalah sebuah bijeksi dari himpunan sisi-sisi pada himpunan bilangan bulat positif $\{1,2, \ldots, q\}$ sedemikian sehingga semua bobot simpul berbeda per-pasangan, dimana bobot simpul dari sebuah simpul adalah jumlah dari label semua sisi yang bersesuaian dengan simpul. Sebuah graf adalah anti-ajaib jika dia mempunyai sebuah pelabelan antiajaib. Dalam paper ini akan dikonstruksi pelabelan anti-ajaib untuk keluarga graf sosis tergeneralisasi.
\end{abstract}

Kata kunci: Pelabelan anti-ajaib, graf sosis tergeneralisasi

\section{INTRODUCTION}

All graphs in this paper are finite, simple, undirected and connected, unless stated otherwise. In 1990, Hartsfield and Ringel [5] introduced the concept of an antimagic labeling of graph, that is, a vertex antimagic edge labeling. An antimagic

2000 Mathematics Subject Classification: 05C78

Received: 24-12-2013, revised: 13-03-2014, accepted: 14-03-2014. 
labeling of a graph $G=(V, E)$ is a bijection $f: E \longrightarrow\{1,2, \ldots,|E|\}$ such that all vertex weights are pairwise distinct, where the weight of a vertex $v$ of $G, w t(v)$, is the sum of the labels of all edges incident with the vertex $v$. A graph $G$ is said to be antimagic if it has an antimagic labeling.

Hartsfield and Ringel [5] showed that $P_{n}, S_{n}, C_{n}, K_{n}, W_{n}$, and $K_{2, n}$, for $n \geq 3$, are antimagic. They also conjectured that every connected graph, except $K_{2}$, is antimagic. Subsequently, several families of graphs have been proved to be antimagic, for example, see $[1,2,3,10]$. Many other results concerning antimagic graphs are catalogued in [4]. Most recently, new families of antimagic graphs have been discovered by Phanalasy et al. [7], Miller et al. [6] and Rylands et al. [9]. However, the conjecture still remains open.

In the previous papers $([7,9]$ for example), the results concerned regular and non regular graphs. Here we are extending the method to cover a class of almost regular graphs. We introduce a new family of graphs, called generalized sausage graphs, and we construct antimagic labeling for such family of graphs. The definition of this family of graphs is stated in Section 2.

Hereafter an edge labeling $l$ of a graph $G$ will be described by an array $L$ (not necessary rectangular), where all edge labels incident with a vertex are written in the same row. Since we are dealing with graphs, each label must occur exactly in two different rows.

\section{Main Results}

We first define a new family of graphs. Let $G$ be a $k$-regular graph with $p$ vertices and $q$ edges. The generalized sausage graph, denoted by $S(G, m)$, is the graph obtained from the Cartesian product graph $G \times P_{m}, m \geq 1\left(G \times P_{1}=G\right)$, by joining each vertex of each end of the $G \times P_{m}$ to a further vertex with an edge; and the two new vertices called apexes. In particular, when $m=1$, each vertex of the graph $G$ joins to two vertices with two edges. The mixed generalized sausage graph, denoted by $M S(G, m)$, is the graph obtained from the generalized sausage graph $S(G, m), m \geq 3$, by joining each vertex of each copy of the $\left\lceil\frac{m}{2}\right\rceil$ copies of $G$ on the left hand side to the left hand side apex, except the nearest copy to the apex, similarly, for the right hand side apex. The complete mixed generalized sausage graph, denoted by $C M S(G, m)$ is the graph obtained from the generalized sausage graph by joining each vertex of each copy of $G$, except the two nearest copies of $G$ to the apexes, to each apex with an edge, and each corresponding pair of vertices of the two nearest copies of $G$ to the apexes with an edge. The complete mixed generalized sausage graph $C M S^{-}(G, m)$ is the graph obtained from $C M S(G, m)$ by deleting the edge connecting each corresponding pair of vertices of the two nearest copies of $G$ to the apexes. For an example of the graph $C M S(G, m)$, see Figure 1. Let $A, B$ and $C$ be the sets of the dark dashed edges, tiny dashed edges and dark edges of the graph in Figure 1, respectively. Then the graph $C M S^{-}\left(C_{3}, 5\right)$ is the 


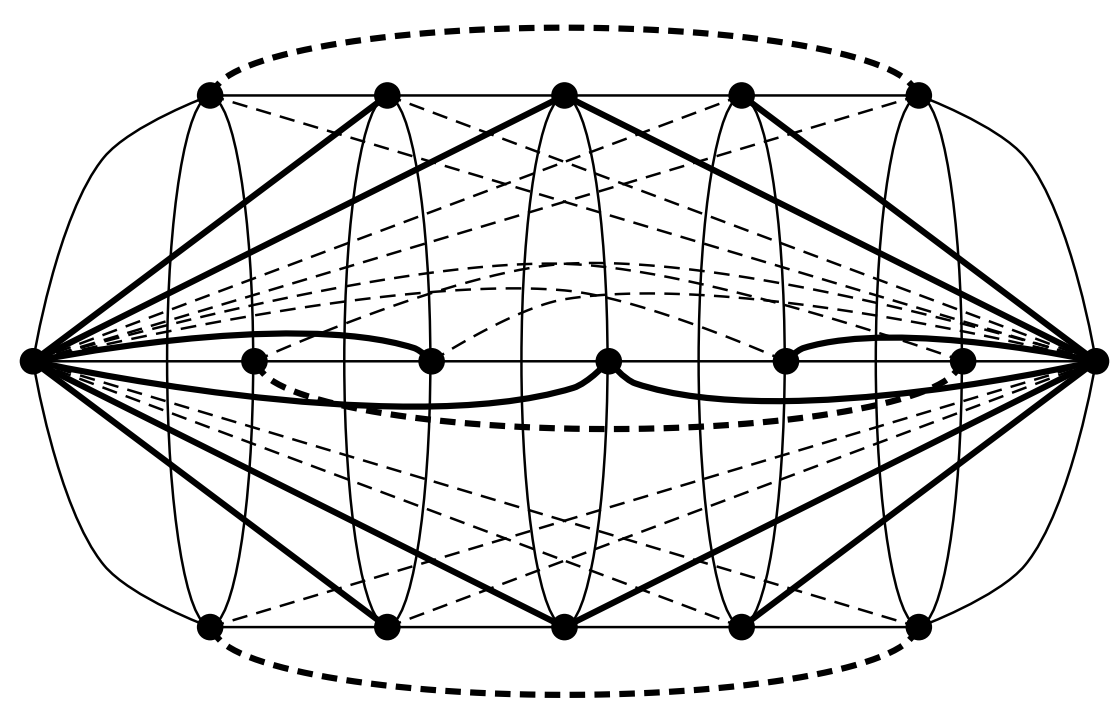

Figure 1. Complete mixed generalized sausage graph $C M S\left(C_{3}, 5\right)$

graph in Figure 1 without $A$, while the graphs $M S\left(C_{3}, 5\right)$ and $S\left(C_{3}, 5\right)$ are the graphs in Figure 1 without $A \cup B$ and without $A \cup B \cup C$, respectively.

Let $G$ be any (connected or diconnected) $k$-regular graph with $p$ vertices and $q$ edges. We first choose any labeling of $G$, that is, label the edges of $G$ by allocating integers $1,2, \ldots q$ randomly. Then calculate the weights of the vertices and order the vertices so that $w t\left(v_{i}\right) \leq w t\left(v_{i+1}\right), 1 \leq i \leq p-1$. This ordering results in an array of edge labels of $G$. It is considered as the original labeling and will be applied throughout the paper to produce antimagic labelings for graphs in the family of generalized sausage graphs.

Denote by $T^{t}$ the transpose of the array $T$. Let $T=\left(\begin{array}{lll}1 & 2 \ldots p-1 & p\end{array}\right)^{t}$. We define the reverse of the array $T$ as $T^{\uparrow}=\left(\begin{array}{lll}p & p-1 \ldots 2 & 1\end{array}\right)^{t}$ and

Theorem 2.1. Let $G \neq n K_{1}, n \geq 1$, be any connected or disconnected $k$-regular graph. Then the generalized sausage graph $S(G, m), m \geq 1$, is antimagic.

Proof. Let $L_{j}, 1 \leq j \leq m$, be the array of edge labels of the $j$-th copy of the graph $G$ in $S(G, m), m \geq 1$. Let $T_{l}, 1 \leq l \leq m+1$, be the $(p \times 1)$-array of the edges $e_{i}, 1 \leq i \leq p$, where $e_{i}$ are the edges of $S(G, m), m \geq 1$, that do not belong to any copy of $G$. We construct the array $A$ of edge labels of $S(G, m), m \geq 1$, as follows.

Case 1: $G=K_{p}, p \geq 2$ 


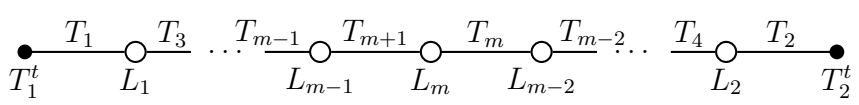

Figure 2. Illustration of antimagic labeling of $S\left(K_{p}, m\right), p \geq$ $2, m \geq 2$ and $m$ even

(1) Label the edge $e_{i}, 1 \leq i \leq p$, in the row $i$ of the array $T_{l}, 1 \leq l \leq m+1$, with $i+(l-1) p$, for $1 \leq l \leq 2$; and $i+(l-1) p+(l-2) q$, for $3 \leq l \leq m+1 ;$

(2) Replace the edge labels in the array $L_{j}, 1 \leq j \leq m$, with new labels obtained by adding $(j+1) p+(j-1) q$ to each of the original edge labels;

(3) Form the array $A$ as shown below.

For $m=1,2$,

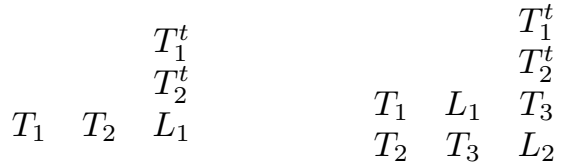

More generally, for $m \geq 3$,

$$
\begin{array}{ccc} 
& & T_{1}^{t} \\
& & T_{2}^{t} \\
T_{1} & L_{1} & T_{3} \\
T_{2} & L_{2} & T_{4} \\
\vdots & \vdots & \vdots \\
T_{m-1} & L_{m-1} & T_{m+1} \\
T_{m} & T_{m+1} & L_{m}
\end{array}
$$

The diagram in Figure 2 illustrates the antimagic labeling used here.

By the construction of the array $A$, it is clear that the weight of each vertex (row) in the array is less than the weight of the vertex (row) below.

Case $2: G \neq K_{p}, p \geq 1$

(1) Replace the edge labels in the array $L_{j}, 1 \leq j \leq m$, with new labels obtained by adding $(j-1)(p+q)$ to each of the original edge labels;

(2) Label the edge $e_{i}, 1 \leq i \leq p$, in the row $i$ of the array $T_{l}, 1 \leq l \leq m+1$, with $i+(l-1) p+l q$, for $1 \leq l \leq m$; and $i+(l-1) p+m q$, for $l=m+1 ;$

(3) Form the array $A$ into two cases as shown below (two separate cases).

Subcase 2.1: $m=1$

$$
\begin{array}{ccc}
L_{1} & T_{1} & T_{2} \\
& & T_{1}^{t} \\
& & T_{2}^{t}
\end{array}
$$


By the construction of the array $A$, it is clear that the weight of each vertex (row) is less than the weight of the vertex (row) below, except the weight of the last row of the subarray $L_{1} T_{1} T_{2}$ and the weight of the row $T_{1}^{t}$ and $T_{2}^{t}$ that needs to be verified. This we do in 3 subcases.

Let $w t\left(r_{f}\right)$ be the weight of the row $r_{f}$.

Subcase 2.1.1: $G=2 K_{2}(p=4, q=2$ and $k=1)$

We have $w t\left(r_{p}\right)=w t\left(T_{1}^{t}\right)=18$. However, by swapping the edge labels 7 and 10 , then all weights of vertices are pairwise distinct.

Subcase 2.1.2: $p=q=4$ and $k=2$

It is simple to check that $w t\left(r_{p}\right)=27, w t\left(T_{1}^{t}\right)=26$ and $w t\left(T_{2}^{t}\right)=42$.

Subcase 2.1.3: $p, q>4, k>1$

Since the largest possible edge labels of the last row in the array $L_{1}$ are $q-(k-1), q-(k-2), \ldots, q-1$ and $q$, hence we have $w t\left(r_{p}\right) \leq(3 p+k+2) q-\frac{k(k-1)}{2}<$ $3 p+(k+2) q<\frac{p(p+1)}{2}+p q=w t\left(T_{1}^{t}\right)$. It is obvious that $w t\left(T_{1}^{t}\right)<w t\left(T_{2}^{t}\right)$.

Subcase 2.2: $m \geq 2$

For $m=2$,

$$
\begin{array}{ccc}
L_{1} & T_{1} & T_{2} \\
T_{1} & L_{2} & T_{3} \\
& & T_{2}^{t} \\
& & T_{3}^{t}
\end{array}
$$

More generally, for $m \geq 3$,

$$
\begin{array}{ccc}
L_{1} & T_{1} & T_{2} \\
T_{1} & L_{2} & T_{3} \\
\vdots & \vdots & \vdots \\
T_{m-2} & L_{m-1} & T_{m} \\
T_{m-1} & L_{m} & T_{m+1} \\
& & T_{m}^{t} \\
& & T^{t}
\end{array}
$$

By the construction of the array $A$, it is clear that the weight of each vertex (row) in the array is less than the weight of the vertex (row) below, except the weights of the last row in the subarray $T_{m-1} L_{m} T_{m+1}$ and the row $T_{m}^{t}$ that need to be verified.

Let $e_{f, g}$ be the edge label in the row $f$ and the column $g$ in the array $A$. We have the largest possible edge labels in the last row (that is the row $r_{m p}$ ) of the array $L_{m}$ and the row $T_{m}^{t}$ as shown below.

$$
\begin{array}{ccccc}
r_{m p}: & \ldots & (q-1)+(m-1)(p+q) & q+(m-1)(p+q) & (m+1) p+m q \\
T_{m}^{t}: & \ldots & m(p+q)-2 & m(p+q)-1 & m(p+q)
\end{array}
$$


We have $e_{m p, p-2}+e_{m p, p-1}+e_{m p, p} \leq(3 m-1) p+3 m q-1 \leq 3 m p+3 m q-3=$ $e_{m p+1, p-2}+e_{m p+1, p-1}+e_{m p+1, p}$. Since $e_{m p, g}<e_{m p+1, g}$ (in case there is no $e_{m p, g}$, we assume $\left.e_{m p, g}=0\right), 1 \leq g \leq p-2$ and $p \geq 2$, therefore $w t\left(r_{m p}\right)<w t\left(T_{m}^{t}\right)$.

When the array $L_{j}, 1 \leq j \leq m$, is removed from the construction given in the proof of Case 1 of Theorem 2.1, the sausage graph degenerates into a path and so gives an alternative proof of the path being antimagic. The path has been proved to be antimagic originally in $[5,8]$.

Corollary 2.2. The generalized sausage graph $S\left(K_{1}, m\right)=P_{m+2}, m \geq 1$, is antimagic.

Corollary 2.3. The generalized sausage graph $S\left(n K_{1}, m\right), m \geq 1$ and $n \geq 2$, is antimagic.

Proof. For $n=2, S\left(2 K_{1}, m\right)$ is a circle $C_{2 m+2}$. It has been proved to be antimagic in $[5,8]$.

We next prove it for $n \geq 3$. We first label the edges of the path $P_{m+2}$ as shown in the diagram in Figure 3. We label that the edge $e_{i}, 1 \leq i \leq m+1$, of $P_{m+2}$ labels with $i$. This ensures that the weights of the vertices with degree 2 are pairwise distinct. To build the graph $S\left(n K_{1}, m\right)$ we use $n$ copies of $P_{m+2}$. Let $L_{j}$, $1 \leq j \leq n$, be the array of $j$-th copy of $P_{m+2}$, where the weights of the vertices with degree 2 are in the ascending order. We construct the array $A$ of edge labels of $S\left(n K_{1}, m\right), m \geq 1$ and $n \geq 2$, as follows.

(1) Replace the label $i$ of the edge $e_{i}$ in the array $L_{j}, 1 \leq j \leq n$, by adding $(j-1)(m+1)$ to each of the original edge labels;

(2) Form the array $A$ as shown below.

$$
\begin{gathered}
L_{1}^{\prime} \\
L_{2}^{\prime} \\
\vdots \\
L_{n}^{\prime} \\
A_{1} \\
A_{2}
\end{gathered}
$$

where $L_{j}^{\prime}, 1 \leq j \leq n$, is the array of the edge labels of the vertices of degree 2 of $j$ th-copy of $P_{m+2}, A_{1}=\left(\begin{array}{ll}m & m+(m+1) \\ 1\end{array}\right)+2(m+1) \ldots m+(n-$ 1) $(m+1))$ and $A_{2}=\left(\begin{array}{llll}m+1 & 2(m+1) & 3(m+1) \ldots n(m+1)\end{array}\right)$.

We skip details of the proof when $n \geq 3$ and $m=1$. For the case $n \geq 3$ and $m=2$, we only need a small change from the case of $n=3$ and $m=2$ by swapping the labels 1 and 2; and the rest of the proof is skipped here since it is similar to the following case.

We now consider the case $n \geq 3$ and $m \geq 3$. By the construction of the array $A$, it clear that the weight of each vertex (row) in the array is less than the weight 


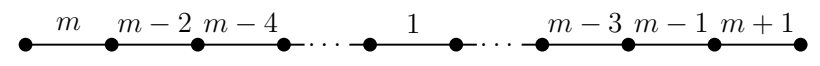

Figure 3. Illustration of a labeling of the path $P_{m+2}, m \geq 1$

of the vertex (row) below, except the weight of the last four rows that need to be verified.

Let $r_{n m-1}$ and $r_{n m}$ be the last two rows of the subarray $L_{n}^{\prime}$. We have $w t\left(r_{n m-1}\right)=m+(n-1)(m+1)+(m-2)+(n-1)(m+1)=2(n+1) m-4$, $w t\left(r_{n m}\right)=n(m+1)+(m-1)+(n-1)(m+1)=2 n(m+1)-2, w t\left(A_{1}\right)=$ $n m+\frac{n(n-1)(m+1)}{2}$ and $w t\left(A_{2}\right)=\frac{n(n+1)(m+1)}{2}$.

For $n=3$, we have $w t\left(r_{n m-1}\right)=6 m+2, w t\left(r_{n m}\right)=6 m+4, w t\left(A_{1}\right)=6 m+3$ and $w t\left(A_{2}\right)=6 m+6$.

For $n \geq 4$, we have $w t\left(A_{1}\right)-w t\left(r_{n m}\right)=\frac{n^{2} m-3 n m+n^{2}-5 n+4}{2}>0$. Therefore, $w t\left(r_{n m-1}\right)<w t\left(r_{n m}\right)<w t\left(A_{1}\right)<w t\left(A_{2}\right)$.

We extend Theorem 2.1 to more general cases in the following theorems and corollaries.

Theorem 2.4. Let $G \neq n K_{1}, n \geq 1$, be any connected or disconnected $k$-regular graph. Then the mixed generalized sausage graph $M S(G, m), m \geq 3$, is antimagic.

Proof. Let $L_{j}, 1 \leq j \leq m$, be the array of edge labels of the $j$-th copy of the graph $G$ in $M S(G, m), m \geq 3$. We construct the array $A$ of edge labels of $M S(G, m)$, $m \geq 3$, as follows.

Case 1: $m \geq 3$ and $m$ odd

Let $T_{l}, 1 \leq l \leq 2 m$, be the $(p \times 1)$-array of the edges $e_{i}, 1 \leq i \leq p$, where $e_{i}$ are the edges of $M S(G, m), m \geq 3$, that do not belong to any copy of $G$.

Subcase 1.1: $m=3$

First consider $K_{p}$ and then other graphs.

Subcase 1.1.1: $G=K_{p}, p \geq 2$

(1) Label the edges $e_{i}, 1 \leq i \leq p$, in the row $i$ of the array $T_{l}, 1 \leq l \leq 2 m$, with $i+(l-1) p$

(2) Replace the edge labels in the array $L_{j}, 1 \leq j \leq m$, with new labels obtained by adding $2 m p+(j-1) q$ to each of the original edge labels;

(3) Form the array $A$ as shown below.

$$
\begin{array}{ccccc} 
& & & T_{1}^{t} & T_{3}^{t} \\
& & & T_{2}^{t} & T_{4}^{t} \\
& & T_{3} & T_{5} & L_{1} \\
& & T_{4} & T_{6} & L_{2} \\
T_{1} & T_{2} & T_{5} & T_{6} & L_{3}
\end{array}
$$


By the construction of the array $A$, it is clear that the weight of each vertex (row) in the array is less than the weight of the vertex (row) below, except possibly for some special cases below.

Let $w t\left(r_{f}\right)$ be the weight of the row $r_{f}$.

(a) $T_{2}^{t} T_{4}^{t}$ and the first row of $T_{3} T_{5} L_{1}$

Since the least possible edge labels (that yield the least possible weight) of the vertex in the array $L_{1}$ are $1+6 p, 2+6 p, \ldots, k+6 p$, hence $w t\left(r_{3}\right)>(1+2 p)+$ $(1+4 p)+(1+6 p)+(2+6 p)+\cdots+(k+6 p)=6 p^{2}+\frac{p^{2}-p}{2}+2>5 p^{2}+p=w t\left(T_{2}^{t} T_{4}^{t}\right)$.

(b) Last row of $T_{4} T_{6} L_{2}$ and the first row of $T_{1} T_{2} T_{5} T_{6} L_{3}$

Let $r_{2 m+2}$ and $r_{2 m+3}$ be the last row of the array $T_{4} T_{6} L_{2}$ and the first row of the array $T_{1} T_{2} T_{5} T_{6} L_{3}$, respectively. We have the edge labels of the rows $r_{2 m+2}$ and $r_{2 m+3}$ as shown below.

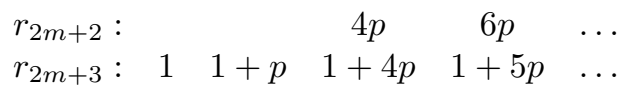

Since $4 p+6 p=10 p<10 p+4=1+(1+p)+(1+4 p)+(1+5 p)$ and all edge labels in the array $L_{2}$ are less than the least possible edge label in the array $L_{3}$, hence $w t\left(r_{2 m+2}\right)<w t\left(r_{2 m+3}\right)$.

Subcase 1.1.2: $G \neq K_{p}, p \geq 1$

(1) Replace the edge labels in the array $L_{j}, 1 \leq j \leq m$, with new labels obtained by adding $(j-1) q$ to each of the original edge labels;

(2) Label the edges $e_{i}, 1 \leq i \leq p$, in the row $i$ of the array $T_{l}, 1 \leq l \leq 2 m$, with $i+(l-1) p+m q$

(3) Form the array $A$ as shown below.

$$
\begin{aligned}
& \begin{array}{lll}
L_{1} & T_{1} & T_{3}
\end{array} \\
& \begin{array}{lll}
L_{2} & T_{2} & T_{4}
\end{array} \\
& \begin{array}{lllll}
L_{3} & T_{3} & T_{4} & T_{5} & T_{6}
\end{array} \\
& \begin{array}{ll}
T_{1}^{t} & T_{5}^{t} \\
T_{2}^{t} & T_{6}^{t}
\end{array}
\end{aligned}
$$

By the construction of the array $A$, it is clear that the weight of each vertex (row) in the array is less the weight of the vertex (row) below, except the weights of the last row of the subarray $L_{3} T_{3} T_{4} T_{5} T_{6}$ and the row $T_{1}^{t} T_{5}^{t}$ that need to be verified.

Let $r_{3 m}$ and $r_{3 m+1}$ be the last row of the subarray $L_{3} T_{3} T_{4} T_{5} T_{6}$ and the row $T_{1}^{t} T_{5}^{t}$, respectively. Since $p \geq k+2$, we have the edge labels of the rows $r_{3 m}$ and $r_{3 m+1}$ as shown below.

$$
\begin{array}{cccccc}
r_{3 m}: & \ldots & 3 p+3 q & 4 p+3 q & 5 p+3 q & 6 p+3 q \\
r_{3 m+1}: & \ldots & 5 p+3 q-3 & 5 p+3 q-2 & 5 p+3 q-1 & 5 p+3 q
\end{array}
$$

We have $(3 p+3 q)+(4 p+3 q)+(5 p+3 q)+(6 p+3 q)=18 p+12 q<20 p+12 q-6=$ $(5 p+3 q-3)+(5 p+3 q-2)+(5 p+3 q-1)+(5 p+3 q)$, for $p>3$, and the largest possible of the rest of edge labels in the row $r_{3 m}$ is less than the least edge label of 


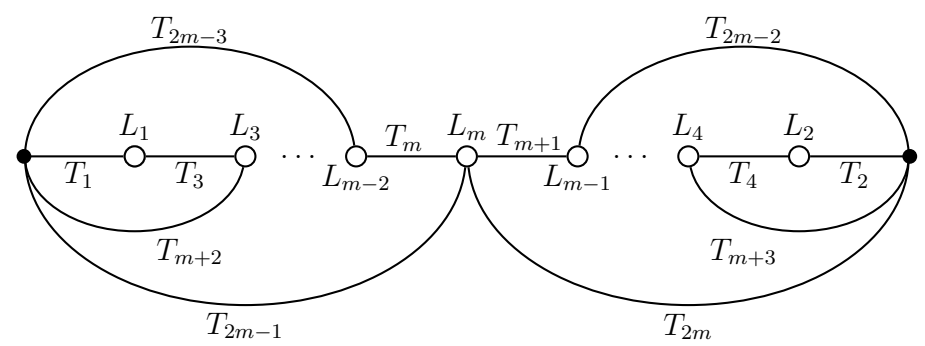

FIGURE 4. Illustration of antimagic labeling of $M S(G, m), m>4$ and $m$ odd

the rest of the edge labels in the row $r_{3 m+1}$, then $w t\left(r_{3 m}\right)<w t\left(r_{3 m+1}\right)$.

Subcase 1.2: $m>4$

(1) Replace the edge labels in the array $L_{j}, 1 \leq j \leq m$, with new labels obtained by adding $(j-1)(p+q)$ to each of the original edge labels;

(2) Label the edges $e_{i}, 1 \leq i \leq p$, in the row $i$ of the array $T_{l}, 1 \leq l \leq 2 m$, with $i+(l-1) p+l q$, for $1 \leq l \leq m$, and $i+(l-1) p+m q$, for $m+1 \leq l \leq 2 m ;$

(3) Form the array $A$ as shown below.

$$
\begin{array}{cccccc} 
& L_{1} & T_{1} & T_{3} & & \\
& L_{2} & T_{2} & T_{4} & & \\
& L_{3} & T_{3} & T_{5} & T_{m+2} & \\
& L_{4} & T_{4} & T_{6} & T_{m+3} & \\
& \vdots & \vdots & \vdots & \vdots & \\
& L_{m-1} & T_{m-1} & T_{m+1} & T_{2 m-2} & \\
& L_{m} & T_{m} & T_{m+1} & T_{2 m-1} & T_{2 m} \\
T_{1}^{t} & T_{m+2}^{t} & \ldots & \ldots & T_{2 m-3}^{t} & T_{2 m-1}^{t} \\
T_{2}^{t} & T_{m+3}^{t} & \ldots & \ldots & T_{2 m-2}^{t} & T_{2 m}^{t}
\end{array}
$$

The diagram in Figure 4 illustrates the antimagic labeling used here.

By the construction of the array, it is clear that the weight of each vertex (row) in the array is less than the weight of the vertex (row) below, except the weights of the last row of the subarray $L_{m} T_{m} T_{m+1} T_{2 m-1} T_{2 m}$ and the row $T_{1}^{t} T_{m+2}^{t} \ldots T_{2 m-5}^{t} T_{2 m-3}^{t} T_{2 m-1}^{t}$ that need to be verified.

Let $r_{m p}$ and $r_{m p+1}$ be the last row of the subarray $L_{m} T_{m} T_{m+1} T_{2 m-1} T_{2 m}$ and the row $T_{1}^{t} T_{m+2}^{t} \ldots T_{2 m-5}^{t} T_{2 m-3}^{t} T_{2 m-1}^{t}$, respectively. We have the edge labels of the rows $r_{m p}$ and $r_{m p+1}$ as shown below.

$$
\begin{array}{ccccc}
r_{m p}: & \ldots & (m+1) p+m q & (2 m-1) p+m q & 2 m p+m q \\
r_{m p+1}: & \ldots & (2 m-1) p+m q-2 & (2 m-1) p+m q-1 & (2 m-1) p+m q
\end{array}
$$

We have $(m+1) p+m q+(2 m-1) p+m q+2 m p+m q=5 m p+3 m q<$ $(6 m-3) p+3 m q-3=(2 m-1) p+m q-2+(2 m-1) p+m q-1+(2 m-1) p+m q$ 
and the rest of the edge labels in the row $r_{m p}$ is less than the edge label in the row $r_{3 m+1}$ of the corresponding column. Hence $w t\left(r_{m p}\right)<w t\left(r_{m p+1}\right)$.

Case 2: $m \geq 4$ and $m$ even

Let $T_{l}, 1 \leq l \leq 2 m-1$, be the $(p \times 1)$-array of the edges $e_{i}, 1 \leq i \leq p$, where $e_{i}$ are the edges of $M S(G, m), m \geq 3$, that do not belong to any copy of $G$.

(1) Replace the edge labels in the array $L_{j}, 1 \leq j \leq m$, with new labels by adding $(j-1) q$ to each of the original edge labels;

(2) Label the edges $e_{i}, 1 \leq i \leq p$, in the row $i$ of the array $T_{l}, 1 \leq l \leq 2 m-1$, with $i+(l-1) p+m q$;

(3) Form the array $A$ into two cases as shown below.

Subcase 2.1: $m=4$

$$
\begin{array}{llll}
L_{1} & T_{1} & T_{3} & \\
L_{2} & T_{2} & T_{4} & \\
L_{3} & T_{3} & T_{5} & T_{6} \\
L_{4} & T_{4} & T_{6} & T_{7} \\
& & T_{1}^{t} & T_{5}^{t} \\
& & T_{2}^{t} & T_{7}^{t}
\end{array}
$$

For $G=K_{2}$, we swap the labels 17 and 18 in the array $T_{7}$.

For $q \geq p$, by the construction of the array $A$, it is clear that the weight of each vertex (row) is less than the weight of the vertex (row) below, except the last row of the subar ray $L_{4} T_{4} T_{6} T_{7}$ and the row $T_{1}^{t} T_{5}^{t}$ that need to be verified.

Let $e_{f, g}$ be the edge label in the row $f$ and the column $g$. Let $r_{4 p}$ and $r_{4 p+1}$ be the last row of subarray $L_{4} T_{4} T_{6} T_{7}$ and the row $T_{1}^{t} T_{5}^{t}$, respectively. We have the edge labels in the rows $r_{4 p}$ and $r_{4 p+1}$ as shown below.

$$
\begin{array}{ccccc}
r_{4 p}: & & \ldots & 6 p+4 q & 7 p+4 q \\
r_{4 p+1}: & 1+4 q & \ldots & 5 p+4 q-1 & 5 p+4 q
\end{array}
$$

Since $e_{4 p, 2 p-1}+e_{4 p, 2 p}=(6 p+4 q)+(7 p+4 q)=13 p+8 q<10 p+12 q=$ $(1+4 q)+(5 p+4 q-1)+(5 p+4 p)=e_{4 p+1,1}+e_{4 p+1,2 p-1}+e_{4 p+1,2 p}$ and $e_{4 p, g}<e_{4 p+1, g}$, for $2 p-(k+2) \leq g \leq 2 p-2$, hence $w t\left(r_{4 p}\right)<w t\left(r_{4 p+1}\right)$. 


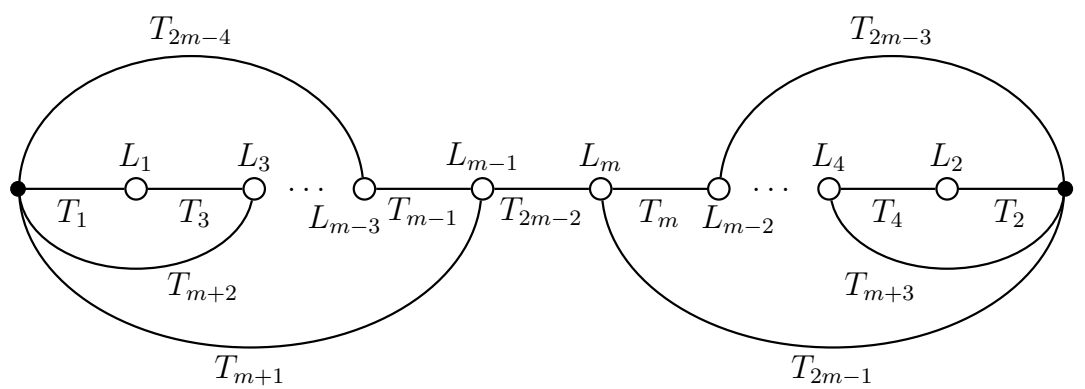

FiguRE 5. Illustration of antimagic labeling of $M S(G, m), m>4$ and $m$ even

Subcase 2.2: $m \geq 6$

\begin{tabular}{|c|c|c|c|c|}
\hline & $L_{1}$ & $T_{1}$ & $T_{3}$ & \\
\hline & $L_{2}$ & $T_{2}$ & $T_{4}$ & \\
\hline & $L_{3}$ & $T_{3}$ & $T_{5}$ & $T_{m+2}$ \\
\hline & $L_{4}$ & $T_{4}$ & $T_{6}$ & $T_{m+3}$ \\
\hline & $\vdots$ & $\vdots$ & $\vdots$ & $\vdots$ \\
\hline & $L_{m-1}$ & $T_{m-1}$ & $T_{m+1}$ & $T_{2 m-2}$ \\
\hline & $L_{m}$ & $T_{m}$ & $T_{2 m-2}$ & $T_{2 m-1}$ \\
\hline$T_{m+1}^{t}$ & $\ldots$ & $\ldots$ & $T_{2 m-6}^{t}$ & $T_{2 m-4}^{t}$ \\
\hline$T_{m+3}^{t}$ & $\ldots$ & $\ldots$ & $T_{2 m-3}^{t}$ & $T_{2 m-1}^{t}$ \\
\hline
\end{tabular}

The diagram in Figure 5 illustrates the antimagic labeling used here.

By the construction of the array $A$, it is clear that the weight of each vertex (row) is less than the weight of the vertex (row) below, except the last row of the subarray $L_{m} T_{m} T_{2 m-2} T_{2 m-1}$ and the row $T_{1}^{t} T_{m+1}^{t} \ldots T_{2 m-8}^{t} T_{2 m-6}^{t}$ $T_{2 m-4}^{t}$ that need to be verified.

Let $e_{f, g}$ be the edge label in the row $f$ and the column $g$. Let $r_{m p}$ and $r_{m p+1}$ be the last row of subarray $L_{m} T_{m} T_{2 m-2} T_{2 m-1}$ and the row $T_{1}^{t} T_{m+1}^{t} \ldots T_{2 m-8}^{t} T_{2 m-6}^{t}$ $T_{2 m-4}^{t}$, respectively. We have the greatest possible labels of the row $r_{m p}$ (when the last row of $L_{m}$ has the largest labels) and the labels of the $r_{m p+1}$ as shown below. We consider in three cases.

(a) $m=6$

$$
\begin{array}{ccccccc}
r_{m p}: & & \ldots & m q & m(p+q) & (2 m-2) p+m q & (2 m-1) p+m q \\
r_{m p+1}: & 1+m q & \ldots & a-3 & a-2 & a-1 & a
\end{array}
$$

where $a=(2 m-4) p+m q$.

Since, for $p \geq 2$, we have $\Sigma_{h=0}^{3} e_{m p, \frac{m}{2} p-h}=m q+m(p+q)+((2 m-2) p+$ $m q)+((2 m-1) p+m q)=(5 m-3) p+4 m q<4(2 m-4) p+4 m q-6=$ $a+(a-1)+(a-2)+(a-3)=\Sigma_{h=0}^{3} e_{m p+1, \frac{m}{2} p-h} ;$ and $e_{m p, g}<e_{m p+1, g}$, for $\frac{m}{2} p-(k+2) \leq g \leq \frac{m}{2} p-4$, hence $w t\left(r_{m p}\right)<w t\left(r_{m p+1}\right)$. 
(b) $m \geq 8$ and $p=2$

$$
\begin{array}{ccccccc}
r_{m p}: & & \ldots & m q & m(p+q) & (2 m-2) p+m q & (2 m-1) p+m q \\
r_{m p+1}: & 1+m q & \ldots & b-1 & b & a-1 & a
\end{array}
$$

where $a=(2 m-4) p+m q$ and $b=(2 m-6) p+m q$.

Similarly to (a), we have $w t\left(r_{m p}\right)<w t\left(r_{m p+1}\right)$.

(c) $m \geq 8$ and $p \geq 3$

It follows immediately from $(\mathrm{b})$, hence $w t\left(r_{m p}\right)<w t\left(r_{m p+1}\right)$.

We give an example for the construction in the proof of Theorem 2.4 (Subcase 1.2) in Figure 6.

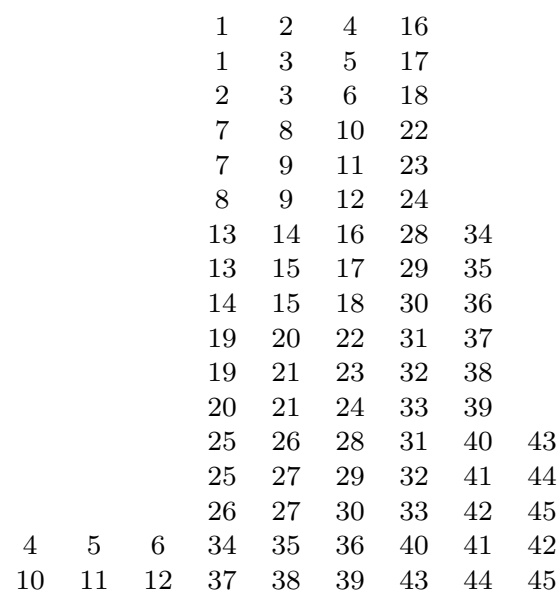

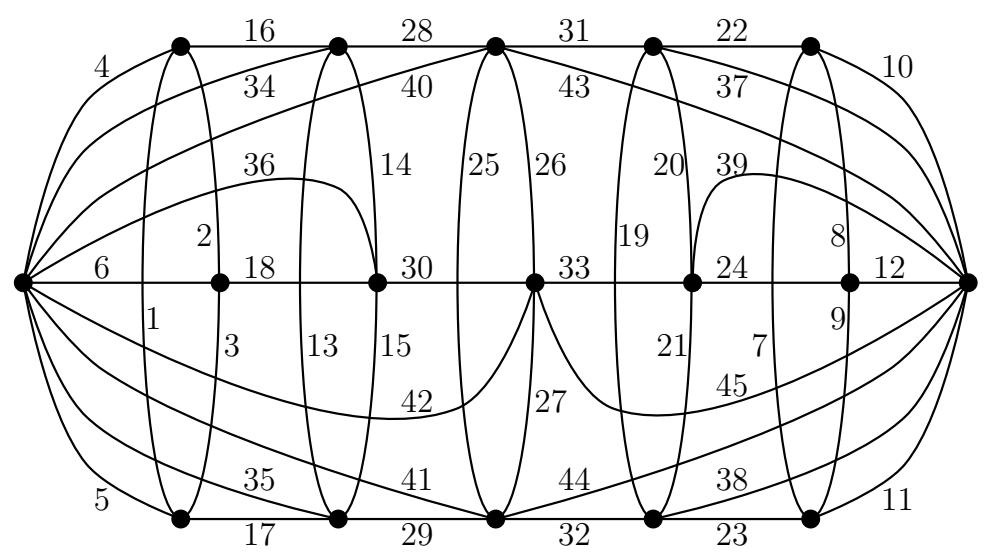

Figure 6. Antimagic labling of $M S\left(C_{3}, 5\right)$ 
Corollary 2.5. The mixed generalized sausage graph $M S\left(K_{1}, m\right), m \geq 3$, is antimagic.

Proof. We divide the proof into three cases.

Case 1: $m=3$

The same construction as the one given in the proof of Subcase 1.1.1 of Theorem 2.4 also works whenever the array $L_{j}, 1 \leq j \leq 3$, is removed.

Case 2: $m \geq 4$

Subcase 2.1: $m$ is odd

The same construction as the one given in the proof of Subcase 1.2 of Theorem 2.4 also works whenever the array $L_{j}, 1 \leq j \leq m$, is removed.

Subcase 2.2: $m$ is even

The same construction as the one given in the proof of Case 2 of Theorem 2.4 also works whenever the array $L_{j}, 1 \leq j \leq m$, is removed. Using this construction, when $m=4$ and $m=6$, there are some weights are equal. However, we need only a small change by swapping the labels 5 and 6 , then all vertex weights are pairwise distinct.

Corollary 2.6. The mixed generalized sausage graph $M S\left(n K_{1}, m\right), m \geq 3$ and $n \geq 2$, is antimagic.

Proof. For $m$ odd, the same constructions as the one given in the proof of Subcase 1.1.2 and Subcase 1.2 of Theorem 2.4 also work whenever the array $L_{j}$, $1 \leq j \leq m$, is removed.

For $m=4$, the same construction as the one given in the proof of Subcase 2.1 of Theorem 2.4 also works whenever the array $L_{j}, 1 \leq j \leq m$, is removed; except when $n=2$, we need a small change by swapping 13 and 14 , and when $n=3$ by swapping 20 and 21 .

For $m \geq 6$ and $m$ even, the same construction as the one given in the proof of Case 2.2 of Theorem 2.4 also works whenever the array $L_{j}, 1 \leq j \leq m$, is removed.

Recall the definition of the reverse $T^{\uparrow}$ from Section 2; we will use it in the proofs of the following theorems and corollaries.

Theorem 2.7. Let $G \neq n K_{1}, n \geq 1$, be any connected or disconnected $k$-regular graph. Then the complete mixed generalized sausage graph $C M S(G, m), m \geq 3$, is antimagic.

Proof. Let $L_{j}, 1 \leq j \leq m$, be the array of edge labels of the $j$-th copy of the graph $G$ in $C M S(G, m), m \geq 3$. Let $T_{l}, 1 \leq l \leq 3 m$, be the $(p \times 1)$-array of the edges $e_{i}, 1 \leq i \leq p$, where $e_{i}$ are the edges of $C M S(G, m), m \geq 3$, that do not belong to any copy of $G$. We construct the array $A$ of $C M S(G, m), m \geq 3$, as follows. 


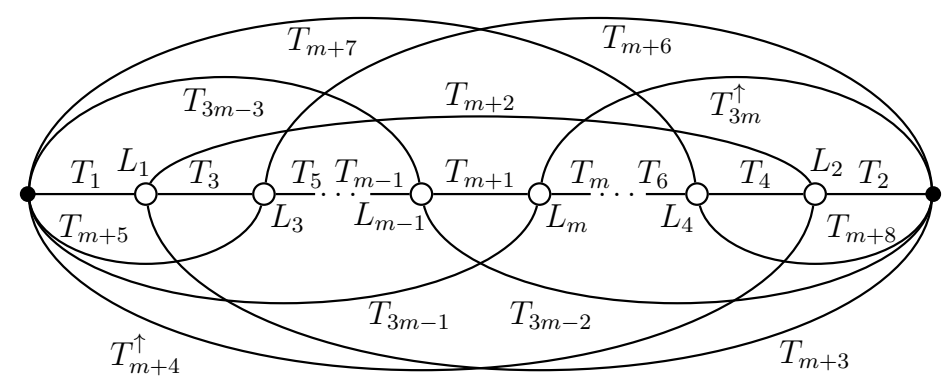

FigURE 7. Illustration of antimagic labeling of $C M S(G, m), m \geq$ 3 and $m$ even

(1) Replace the edge labels in the array $L_{j}, 1 \leq j \leq m$, with new labels obtained by adding $(j-1)(p+q)$ to each of the original edge labels;

(2) Label the edges $e_{i}, 1 \leq i \leq p$, in the row $i$ of the array $T_{l}, 1 \leq l \leq 3 m$, with $i+(l-1) p+l q$, for $1 \leq l \leq m$, and $i+(l-1) p+m q$, for $m+1 \leq l \leq 3 m$;

(3) Form the array $A$ as shown below.

For $m=3,4$,

$$
\begin{array}{cccccccccc}
L_{1} & T_{1} & T_{3} & T_{5} & T_{6} & L_{1} & T_{1} & T_{3} & T_{5} & T_{6} \\
L_{2} & T_{2} & T_{4} & T_{5} & T_{7}^{\uparrow} & L_{2} & T_{2} & T_{4} & T_{6} & T_{8}^{\uparrow} \\
L_{3} & T_{3} & T_{4} & T_{8} & T_{9}^{\uparrow} & L_{3} & T_{3} & T_{5} & T_{9} & T_{10} \\
& & T_{1}^{t} & T_{7}^{t} & T_{8}^{t} & L_{4} & T_{4} & T_{5} & T_{11} & T_{12}^{\uparrow} \\
& & T_{2}^{t} & T_{6}^{t} & T_{9}^{t} & & T_{1}^{t} & T_{8}^{t} & T_{9}^{t} & T_{11}^{t} \\
& & & & & & T_{2}^{t} & T_{7}^{t} & T^{t} 11 & T_{12}^{t}
\end{array}
$$

More generally, for $m>4$,

$$
\begin{aligned}
& L_{1} \quad T_{1} \quad T_{3} \quad T_{m+2} \quad T_{m+3} \\
& L_{2} \quad T_{2} \quad T_{4} \quad T_{m+2} \quad T_{m+4}^{\uparrow} \\
& \begin{array}{lllll}
L_{3} & T_{3} & T_{5} & T_{m+5} & T_{m+6}
\end{array} \\
& \begin{array}{lllll}
L_{4} & T_{4} & T_{6} & T_{m+7} & T_{m+8}
\end{array} \\
& L_{m-2} \quad T_{m-2} \quad T_{m} \quad T_{3 m-5} \quad T_{3 m-4} \\
& L_{m-1} \quad T_{m-1} \quad T_{m+1} \quad T_{3 m-3} \quad T_{3 m-2} \\
& \begin{array}{lllll}
L_{m} & T_{m} & T_{m+1} & T_{3 m-1} & T_{3 m}^{\uparrow}
\end{array} \\
& \begin{array}{llllllll}
T_{1}^{t} & T_{m+4}^{t} & T_{m+5}^{t} & \ldots & \ldots & T_{3 m-5}^{t} & T_{3 m-3}^{t} & T_{3 m-1}^{t}
\end{array} \\
& \begin{array}{llllllll}
T_{2}^{t} & T_{m+3}^{t} & T_{m+6}^{t} & \ldots & \ldots & T_{3 m-4}^{t} & T_{3 m-2}^{t} & T_{3 m}^{t}
\end{array}
\end{aligned}
$$

The diagram in Figure 7 illustrates the antimagic labeling used here. 
By the construction of the array $A$, it clear that the weight of each vertex (row) is less than the weight of the vertex (row) below, except some special cases that need to be verified.

We skip details for the case $m=3$ and next verify for the case $m \geq 4$.

Let $e_{f, g}$ be the label at the row $f$ and the column $g$ in the array $A$.

(a) Rows $r_{p}$ and $r_{p+1}$

We have the edge labels of rows $r_{p}$ and $r_{p+1}$ as shown.

$$
\begin{array}{cccc}
r_{p}: & \ldots & (m+2) p+m q & (m+3) p+m q \\
r_{p+1}: & \ldots & 1+(m+1) p+m q & (m+4) p+m q
\end{array}
$$

Since $e_{p, m p-1}+e_{p, m p}=(2 m+5) p+2 m q<(2 m+5) p+2 m q+1$, for all $p$ and $q$, and $e_{p, g}<e_{p+1, g}$, for $1 \leq g \leq m p-2$, hence $w t\left(r_{p}\right)<w t\left(r_{p+1}\right)$.

(b) Rows $r_{(m-1) p}$ and $r_{(m-1) p+1}$

We have $e_{(m-1) p, m p-2}+e_{(m-1) p, m p}=(4 m-1) p+2 m q<4 m p+2 m q+1=$ $e_{(m-1) p+1, m p-2}+e_{(m-1) p+1, m p}$, for all $p$ and $q$; and $e_{(m-1) p, g}<e_{(m-1) p+1, g}$, for $1 \leq g \leq m p-3$, and $g=m p-1$. Then $w t\left(r_{(m-1) p}\right)<w t\left(r_{(m-1) p+1}\right)$.

(c) Rows $r_{m p}$ and $r_{m p+1}$

Since $m \geq 4$ and $p \geq 2$, it is clear that $r_{m p}<r_{m p+1}$.

(d) Rows $r_{m p+1}$ and $r_{m p+2}$

Let $A$ and $B$ be the sum of all the edge labels in subarrays $T_{1}^{t} T_{m+4}^{t}$ and $T_{2}^{t} T_{m+3}^{t}$, respectively. It is easy to check that $A<B$ and $e_{m p+1, g}<e_{m p+2, g}$, for $2 p+1 \leq g \leq m p$. Hence $w t\left(r_{m p+1}\right)<w t\left(r_{m p+2}\right)$.

The same construction as the one given in the proof of Theorem 2.7 also works when the array $L_{j}, 1 \leq j \leq m$, is removed. We have

Corollary 2.8. The complete mixed generalized sausage graph $C M S\left(n K_{1}, m\right)$, $m \geq 3, n \geq 1$, is antimagic.

See Section 2 for definition of $C M S^{-}(G, m)$, then we have

Corollary 2.9. Let $G \neq n K_{1}, n \geq 1$, be any connected or disconnected $k$-regular graph. Then the complete mixed generalized sausage graph $C M S^{-}(G, m), m \geq 2$, is antimagic.

Proof. If $m \geq 3$, remove $T_{m+2}$ from the array $A$ in the proof of Theorem 2.7 and replace the array $T_{l}$ with $T_{l-1}$, for $m+3 \leq l \leq 3 m$.

If $m=2$, we construct the array $A$ as shown.

$$
\begin{array}{cccc}
L_{1} & T_{1} & T_{2} & T_{3} \\
L_{2} & T_{1} & T_{4} & T_{5}^{\uparrow} \\
& & T_{2}^{t} & T_{4}^{t} \\
& & T_{3}^{t} & T_{5}^{t}
\end{array}
$$

For $p=2$, that is, $G=K_{2}$, it is easy to check that $C M S^{-}\left(K_{2}, 2\right)$ is antimagic. 
For $p \geq 3$, it is similar to (a) in the proof of Theorem 2.7 for checking $w t\left(r_{p}\right)<w t\left(r_{p+1}\right)$. We next prove that $w t\left(r_{2 p}\right)<w t\left(r_{2 p+1}\right)$. We have the edge labels of $r_{2 p}$ and $w t\left(r_{2 p+1}\right)$ as shown.

$$
\begin{array}{ccccc}
r_{2 p}: & \ldots & p+q & 4 p+3 q & 1+4 p+3 q \\
r_{2 p+1}: & \ldots & 4 p+3 q-2 & 4 p+3 q-1 & 4 p+3 q
\end{array}
$$

Since $e_{2 p, 2 p-2}+e_{2 p, 2 p-1}+e_{2 p, 2 p}=9 p+7 q+1<12 p+9 p-3=e_{2 p+1,2 p-2}+$ $e_{2 p+1,2 p-1}+e_{2 p+1,2 p}$ and $e_{2 p, g}<e_{2 p+1, g}$, for $2 p-k-2 \leq g \leq 2 p-3$, hence $w t\left(r_{2 p}\right)<w t\left(r_{2 p+1}\right)$.

The same construction as the one given in Corollary 2.9 also works when the array $L_{j}, 1 \leq j \leq m$, is removed, except when $m=2$ and $n=1$, it needs a small change by swapping the labels 1 and 2 . Then we have

Corollary 2.10. The complete mixed generalized sausage graph $C M S^{-}\left(n K_{1}, m\right)$, $m \geq 2, n \geq 1$, is antimagic.

\section{REFERENCES}

[1] Alon, N., Kaplan, G., Lev, A., Roditty, Y. and Yuster, R., "Dense graphs are antimagic", J. Graph Theory, 47(4) (2004), 297-309.

[2] Bača, M. and Miller, M., Super Edge-Antimagic Graphs: A Wealth of Problems and Some Solutions, BrownWalker Press, Boca Raton, Florida, USA, 2008.

[3] Bača, M., Lin, Y. and Semaničová-Feňovčiková, A., "Note on super antimagicness of disconnected graphs", Int. J. Graphs and Comb., 6(1) (2009), 47-55.

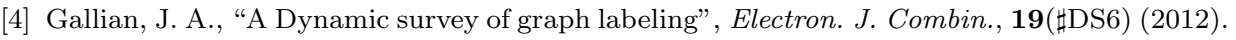

[5] Hartsfield, N. and Ringel, G., Pearls in graph theory: A comprehensive introduction, Academic Press Inc., Boston, MA, 1990.

[6] Miller, M., Phanalasy, O., Ryan, J. and Rylands, L., "Antimagicness of some families of generalized graphs", Austral. J. Combin. 53 (2012), 179-190.

[7] Phanalasy, O., Miller, M., Rylands, L. and Lieby, P., "On a relationship between completely separating systems and antimagic labeling of regular graphs", LNCS 6460 (2011), 238-241.

[8] Ryan, J., Phanalasy, O., Miller, M. and Rylands, L., "On antimagic labeling for generalized web and flower graphs", LNCS 6460 (2011), 303-313.

[9] Rylands, L., Phanalasy, O., Ryan, J. and Miller, M., "An application of completely sepaprating sysrtems to graph labeling", LNCS $\mathbf{8 2 8 8}$ (2013), 376-387.

[10] Zhang, Y. and Sun, X., "The antimagicness of the Cartesian product of graphs", Theoretical Computer Science, 410 (2009), 727-735. 